\title{
Context-specific morphine tolerance and withdrawal: The effects of interdose interval
}

\author{
JULIAN L. AZORLOSA, NICHOLAS E. HARTLEY, and CHERYL DEFFNER-RAPPOLD \\ Southeastern Louisiana University, Hammond, Louisiana
}

\begin{abstract}
In the present experiments, we examined the effect of interdose interval (IDI) on context-specific morphine tolerance and withdrawal in rats. Contextual control of withdrawal was demonstrated with a 48-h IDI and was retained during an 8-day period of drug abstinence. Context-specific tolerance was also acquired and retained with a 48-h IDI. With a $12-\mathrm{h}$ IDI, contextual control of both withdrawal and tolerance was disrupted. These studies demonstrated context-specific withdrawal with a lower dose $(10 \mathrm{mg} / \mathrm{kg})$ and a longer retention interval than has been previously reported. The finding that both contextual withdrawal and tolerance were similarly affected by the IDI suggests that they are mediated by a common process.
\end{abstract}

A large body of research has established that associative learning plays a major role in the development of tolerance to morphine and other opiates (e.g., Siegel, 1975, 1977, 1978; see Goudie \& Demellweek, 1986, for a review). These studies showed that animals were more tolerant to morphine when it was administered in the presence of contextual cues previously paired with the drug compared with animals that had the same history of morphine exposure paired with different cues. Siegel (1975, 1989) proposed a classical conditioning model of associative tolerance in which the environmental cues present during drug administration serve as a conditioned stimulus (CS) and the drug as the unconditioned stimulus (US). After repeated pairings, the CS elicits compensatory, drug-opposite conditioned responses (CRs), which attenuate the effects of the morphine. Paletta and Wagner (1986) and Baker and Tiffany (1985) view tolerance as a process of habituation that may be mediated by cues paired with morphine. According to Paletta and Wagner, tolerance is also due to compensatory responses that develop to some drug effects.

Repeated administration of morphine also results in dependence, which is characterized by the appearance of withdrawal signs after the drug is discontinued or precipitated by an opiate antagonist. In the rat, these signs include rearing, circling, jumping, wet dog shakes, teeth chattering, and genital licking (Blasig, Hertz, Reinhold, \& Zieglgänsberger, 1973; Linseman, 1977). According to Siegel (1989), withdrawal occurs when the compensatory responses that mediate tolerance are elicited by

This research was supported by a faculty development grant from Southeastern Louisiana University to the first author. The authors thank George A. Cicala for his helpful comments. The morphine sulphate was a gift from the Penick Corp. Correspondence concerning this article should be sent to J. L. Azorlosa, Department of Psychology, SLU 324, Hammond, LA 70402. drug cues in the absence of drug administration. Although some investigators have found evidence of compensatory responses (Krank, Hinson, \& Siegel, 1981; Siegel, 1975, 1978), others have either failed to find evidence of them or have offered alternative explanations for the original demonstrations (see Goudie \& Demellweek, 1986).

Despite the controversy about compensatory responses, several studies have shown that contextual cues can elicit morphine withdrawal signs. Some showed that cues paired with withdrawal sickness elicited withdrawal signs in rats (Wikler \& Pescor, 1967) and hamsters (Schnur, 1992). Other studies have shown that cues repeatedly paired with morphine elicit withdrawal. Kelsey and colleagues, for example, found that morphinedependent rats given a saline injection in the same environment in which they had received morphine displayed more rearing, circling, and genital licking than did rats that had received morphine in another environment (Falls \& Kelsey, 1989; Kelsey, Aranow, \& Matthews, 1990). Hinson and Siegel (1983) demonstrated context-specific circling and jumping, and Krank and Perkins (1993) found contextual control of several withdrawal signs in morphine-dependent rats. However, some recent studies have failed to find contextual control of withdrawal (Sobrero \& Bouton, 1989; Zellner, Dacanay, \& Riley, 1984).

The role of associative learning in opiate withdrawal is of considerable theoretical and practical importance and deserves additional investigation. The present studies had three goals. The first was to demonstrate that cues paired with morphine can elicit contextual withdrawal with a dose lower than that previously reported (Falls \& Kelsey, 1989; Kelsey et al., 1990; Krank \& Perkins, 1993). The second was to determine what effect relatively short intervals between morphine administrations would have on the acquisition (Experiment 1) and 
retention (Experiment 2) of context-specific withdrawal. Previous research has shown that a brief interdose interval (IDI) disrupts context-specific tolerance (Dafters \& Odber, 1989; Kesner \& Cook, 1983; Tiffany \& MaudeGriffin, 1988; Tiffany, Maude-Griffin, \& Drobes, 1991) but there have not been any studies examining the effect of this manipulation on withdrawal. The third goal was to determine whether both contextual withdrawal and tolerance were similarly affected by the IDI manipulation. If they were, this would provide some support for the contention that these phenomena arise from the same process (Siegel, 1975, 1989). In Experiment 1, rats were given discrimination training in which seven injections of morphine were either paired or unpaired with a distinctive environment (DE). Control groups were trained with saline only. Half the rats had training sessions spaced $48 \mathrm{~h}$ apart, and the other half were trained at $12-\mathrm{h}$ intervals. Following the seventh session, tolerance to morphine analgesia was tested in all groups in the DE. Twenty-four hours later, withdrawal was measured in the $\mathrm{DE}$ after a saline injection. It was predicted that context-specific tolerance and withdrawal would be observed in the 48-h IDI, but would be reduced or eliminated in the 12-h IDI.

\section{EXPERIMENT 1}

\section{Method}

\section{Subjects}

The subjects were forty-eight male Sprague-Dawley rats that weighed $240-270 \mathrm{~g}$ at the start of the study. They were housed individually, given ad-lib access to food and water, and maintained on a 15:9-h light:dark cycle. The animals were trained and tested during the light part of the cycle.

\begin{abstract}
Apparatus
Analgesia was measured with a hot plate (Omnitech Model $\mathrm{HP}$ ) set at $52^{\circ} \mathrm{C}$. Training sessions were conducted in the $\mathrm{DE}$, which consisted of two rooms, each containing two cages. One room was lighted with two $25-\mathrm{W}$ red bulbs, one directed at each cage. The walls and tops of the cages in this room were Plexiglas, and the floors were made of stainless steel rods. Each cage was scented with a pine air freshener, and a white noise masked extraneous sounds. The other room was lighted by a partially shaded, 45-W white light. The cages in this room were made of Plexiglas with smooth aluminum floors; they were also scented with pine air freshener. A room fan masked extraneous noise. Between sessions, the cages were cleaned with a pine-scented disinfectant.
\end{abstract}

Drugs. Morphine sulphate $(10 \mathrm{mg} / \mathrm{kg})$ was administered in a $.85 \%$ sodium chloride vehicle in a volume of $1 \mathrm{ml} / \mathrm{kg}$. All injections were subcutaneous in the dorsal neck area.

\section{Procedure}

The rats were handled and pretrained for 3 days prior to the start of the study. On the first pretraining day, the animals were injected with saline; 20 min later, they were placed on the unheated hot plate for $30 \mathrm{sec}$. Hot plate tests (latency to lick a hind paw) were conducted at 40 and $60 \mathrm{~min}$ after the injection. The animals were returned to the home cage for the interval between hot plate tests. On Days 2 and 3, the animals were injected with saline and given three hot plate assessments at 20 -min intervals. The rats remained in the home cage between the tests. Following the hot plate tests, the animals were again injected with saline.

Following pretraining, the rats were randomly assigned to six equal groups. The two paired groups $(\mathrm{P})$ received morphine in the $\mathrm{DE}$ and saline in the home cage. The two unpaired groups (U) received saline in the DE and morphine in the home cage. The two saline groups (S) received saline in both the DE and the home cage. Three of the groups had the sessions spaced $48 \mathrm{~h}$ apart (P48, $\mathrm{U} 48$, and S48), and three had them $12 \mathrm{~h}$ apart (P12, U12, and S12). At the start of a session, the animals were taken to the assigned room, injected, and placed into the assigned cage. At 20,40, and $60 \mathrm{~min}$ postinjection, they were placed onto the unheated hot plate for $30 \mathrm{sec}$. One hour after the injection, they were returned to the home cage. Home cage injections were given $5 \mathrm{~h}$ after the session in the 48-h IDI groups and $2 \mathrm{~h}$ after the session in the 12-h IDI groups. There were seven acquisition sessions. The $48-\mathrm{h}$ groups were run between 0900 and 1700 , and the 12-h groups from 0800 to 1000 and 2000 to 2200 . Each animal was run at approximately the same time during every session. Half of the rats in each group received the sessions in one of the $\mathrm{DE}$ rooms, and half received their sessions in the other room. Assignment to the cages in each room was counterbalanced between groups.

Tolerance testing. Tolerance was assessed on the eighth session and at the same IDI used in acquisition. All the rats were given an injection of morphine in the $\mathrm{DE}$, placed into the appropriate box, and given hot plate tests at 20,40 , and 60 min postinjection. Latencies to lick a hind paw were recorded. The test was terminated after $30 \mathrm{sec}$ if an animal failed to lick. Testing was blind with respect to group assignment.

Withdrawal testing. Twenty-four hours after tolerance testing, each animal was transported to the DE, injected with saline, and placed into the appropriate box. For $10 \mathrm{~min}$, the following withdrawal symptoms were counted: rearing (up on hind feet with body partly or fully extended), genital licking (licking penis), wet dog shakes (brief shake of head, shoulders, and body), and circling (one continuous movement). The observers were blind with respect to the animal's group. Prior to this test, the three observers jointly recorded withdrawal signs in morphine-dependent pilot animals to ensure high interobserver agreement.

Data analysis. The means of the three hot plate scores were calculated for each animal. These scores and the totals for each withdrawal sign were separately analyzed by planned, orthogonal comparisons (Hays, 1981). The four comparisons were (1) the mean of P48 and U48 compared with the mean of S48; (2) the mean of P12 and $\mathrm{U} 12$ compared with the mean of $\mathrm{S} 12$; (3) the mean of $\mathrm{P} 48$ compared with the mean of U48; and (4) the mean of P12 compared with the mean of U12. Comparisons (1) and (2) tested within each IDI, whether prior exposure to morphine produced tolerance (or withdrawal). Comparisons (3) and (4) determined, within each IDI, whether tolerance (or withdrawal) was context specific.

\section{Results}

\section{Tolerance Test}

Inspection of the hot plate scores showed that they were stable during the three test times, so it was appropriate to average them. These data are presented in Figure 1 , which shows that previous exposure to morphine resulted in tolerance. The means of the morphine groups were significantly lower than were their respective saline controls in both the 48-h IDI $[t(42)=4.83, p<.001]$ and the 12 -h IDI $[t(42)=4.32, p<.001]$. It is also apparent that context-specific tolerance occurred only in the 48-h IDI. The latencies of P48 were shorter than 


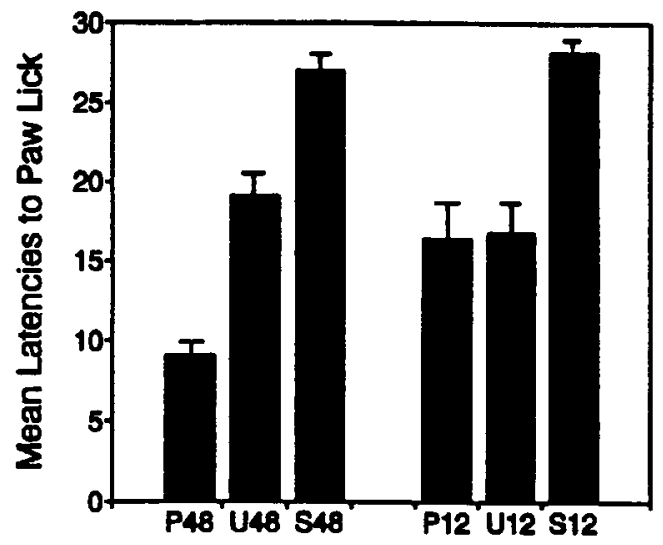

Figure 1. Mean latencies to lick a hind paw during the tolerance test in Experiment 1. P, paired; $U$, unpaired; $S$, saline; 48, 48-h IDI; 12, 12-h IDI. Bars indicate standard error of the mean.

those of $U 48[t(42)=3.24, p<.05]$, but there was no difference between $\mathrm{P} 12$ and $\mathrm{U} 12[t(42)=0.10, p>9]$.

\section{Withdrawal Tests}

Mean number of rears and circles recorded during the $10 \mathrm{~min}$ following a saline injection $(24 \mathrm{~h}$ after the tolerance test) are presented in Figure 2. Rearing (upper panel) was not affected by prior morphine exposure; that is, the comparisons between the mean of the morphine groups and their respective saline controls were not significant in either the 48-h IDI $[t(42)=1.49, p=.072]$ or the 12-h IDI $[t(42)=.172, p>.4]$. Rearing was context specific in the 48 -h IDI $[t(42)=2.91, p<.01]$, but not in the 12-h IDI $[t(42)=1.16, p>.1]$. The bottom panel of Figure 1 shows that the groups trained with the 48-h IDI circled more than did those trained with the 12-h IDI, but none of the comparisons approached significance (all $t \mathrm{~s}<1.0$ ). None of the comparisons for wet dog shakes or genital licks were significant (not shown).

\section{Discussion}

This experiment showed that cues repeatedly paired with morphine can elicit withdrawal at a dose lower than that previously reported (Falls \& Kelsey, 1989; Kelsey et al., 1990; Krank \& Perkins, 1993). It also demonstrated that withdrawal was disrupted by a brief IDI, and it is perhaps the first time that the effect of any conditioning parameter on the acquisition of context-specific withdrawal has been examined. This experiment also confirmed the previous finding (Falls \& Kelsey, 1989) that contextual cues that elicit context-specific withdrawal also mediate analgesic tolerance.

The demonstration of context-specific tolerance in the 48-h IDI, but not in the 12-h IDI, is consistent with other studies showing that a brief IDI attenuated tolerance controlled by contextual cues (Dafters \& Odber, 1989; Kesner \& Cook, 1983; Tiffany \& Maude-Griffin, 1988; Tiffany et al., 1991).
The finding that a brief IDI disrupted both contextspecific tolerance and context-elicited withdrawal is consistent with the model proposed by Siegel (1975, 1989), in which context-specific tolerance and withdrawal both arise from cue-elicited compensatory CRs. Because compensatory responses were not measured, this study only provides tentative support for this idea. Nonetheless, these results suggest a common process for these two phenomena and warrant further investigation.

The purpose of Experiment 2 was to replicate the finding that context-specific withdrawal and tolerance both occur in the 48-h IDI, but are disrupted with a 12-h IDI. The finding is also extended by examining whether context-specific withdrawal and tolerance are retained during a period of drug abstinence. There is abundant evidence for the retention of context-specific tolerance to analgesia (Dafters \& Odber, 1989; Siegel, 1975, 1977; Tiffany et al., 1991), but much less evidence concerning the retention of context-specific withdrawal signs. Schnur (1992) and Wikler and Pescor (1967) demonstrated retention of withdrawal that was elicited by cues paired with withdrawal. Kelsey et al., (1990) found that a context paired with morphine elicited with-
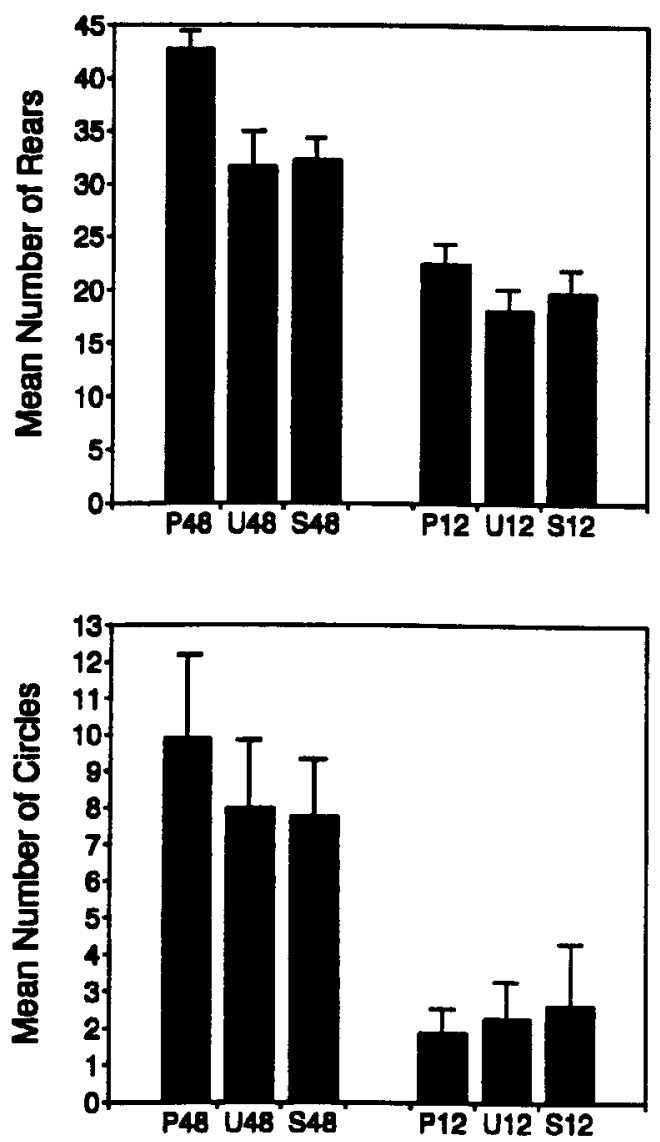

Figure 2. Mean withdrawal scores in Experiment 1. Upper panel, rears; lower panel, circles. 
drawal signs (circles and rears) after a 5-day period of abstinence. Krank and Perkins (1993) found contextspecific withdrawal signs 4 days after the last morphine injection. The final purpose of Experiment 2 was to compare retention of context-specific withdrawal and tolerance. If both are retained, this would provide additional support for the notion that these phenomena arise from a common process.

The procedure used in Experiment 2 was similar to that of Experiment 1. Six groups of rats were given seven morphine administrations in the paired, unpaired, or saline-only conditions, with sessions spaced 48 or $12 \mathrm{~h}$ apart. Tests of withdrawal and tolerance retention were conducted, respectively, after 7 and 8 days of morphine abstinence. Experiment 2 incorporated three minor procedural changes. First, hot plate testing during the pretraining stage was omitted to avoid any preconditioning effects (e.g., Tiffany \& Baker, 1981). Second, two conditioning boxes were added to each DE room. Finally, jumping was measured. This behavior was rarely seen in pilot work done prior to the first study, but was observed in subsequent studies. Jumping was defined as leaping with all four paws off the floor.

\section{EXPERIMENT 2}

\section{Method}

\section{Subjects}

Forty-eight male Sprague-Dawley rats, weighing between 230 and $270 \mathrm{~g}$, were used. They were housed and maintained as in Experiment 1 .

\begin{abstract}
Apparatus
The distinctive rooms and boxes from Experiment 1 were again used. In each room, two additional boxes were used; these were constructed of aluminum, with wire-mesh fronts and smooth wood floors. All the boxes were scented with pine air freshener.

\section{Procedure}

Pretraining was the same as that described previously, except that the hot plate was unheated. The rats were placed on the unheated hot plate and remained there for $30 \mathrm{sec}$.

After pretraining, the rats were randomly assigned to one of six groups (P48, U48, S48, P12, U12, and S12) and given seven tolerance acquisition sessions, as in Experiment 1. Morphine was administered subcutaneously $(10 \mathrm{mg} / \mathrm{kg})$. Tolerance and withdrawal were measured as in Experiment 1. There were two observers, with an interobserver reliability of .98 .

Six days after the first withdrawal test, retention of contextelicited withdrawal was measured. Following a saline injection in the $\mathrm{DE}$, the rats were placed into the assigned box and observed for $10 \mathrm{~min}$. Withdrawal was assessed before tolerance so that the rats would be tested after a long period of drug abstinence. On the day following the withdrawal test, retention of tolerance was measured.
\end{abstract}

\section{Results}

\section{Tolerance Test}

Mean hot plate latencies from the first tolerance test are shown in Figure 3. The combined means of the groups given morphine during acquisition were shorter than were their respective saline controls in both the 48 -h IDI $[t(42)=5.92, p<.001]$ and the 12 -h condition $[t(42)=6.82, p<.001]$. Tolerance was context specific

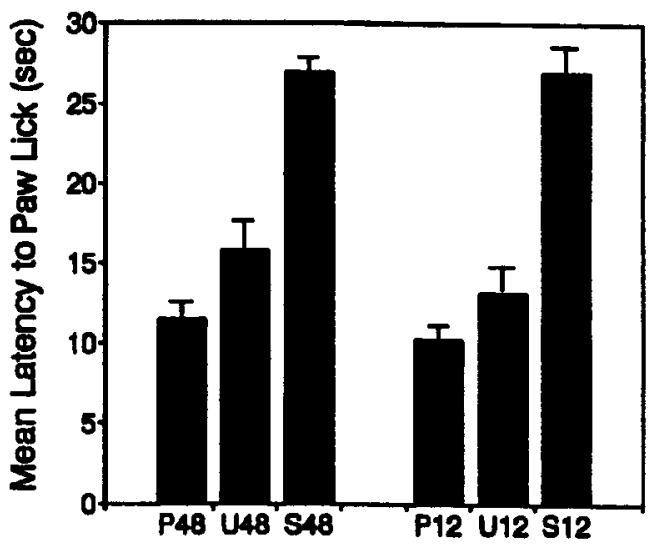

Figure 3. Mean latencies to lick a hind paw during the initial tolerance test in Experiment 2.

only in the 48-h IDI $[t(42)=1.69, p<.05] . \mathrm{P} 12$ and U12 did not differ $[t(42)=1.13, p>.1]$.

\section{Withdrawal Test}

Figure 4 shows the mean number of withdrawal signs recorded during the 10-min period after a saline injection in the DE. The morphine groups reared more (upper panel) than did the saline group in the 48-h IDI $[t(42)=$ $2.42, p<.01]$, but not in the 12-h IDI $[t(42)=1.48, p=$ $.072]$. This effect in the 48-h IDI appears to be due to the high scores of the paired group, and there was an effect of context in both IDI conditions: P48 reared more than $\mathrm{U} 48[t(42)=4.10, p<.001]$, and $\mathrm{P} 12$ reared more than $\mathrm{U} 12[t(42)=1.86, p<.05]$.

Figure 4 (middle and bottom panels, respectively) also shows that in the 48-h IDI, jumping $[t(42)=2.01$, $p<.05]$ and circling $[t(42)=3.56, p<.001]$ were context specific. There were no context effects in the 12-h IDI for either sign $(t \mathrm{~s}<1)$ and no differences between the mean of the morphine groups and the saline control for jumping or circling in either IDI condition $(t s<1)$. None of the comparisons for the other withdrawal signs were significant.

\section{Retention of Withdrawal and Tolerance}

Figure 5 shows three withdrawal signs observed during the 10-min period following a saline injection in the DE 7 days after the last exposure to morphine. The morphine groups reared more than the saline group $[t(42)=$ $1.76, p<.05]$ in the 48-h IDI, but not in the 12-h IDI $(t<1)$. Rearing was context specific in the 48-h condition $[t(42)=1.83, p<.05]$ and the 12 -h IDI $[t(42)=$ $2.46, p<.01]$.

Jumping (middle panel) was context specific only in the 48 -h condition $[t(42)=1.80, p<.05]$. None of the other comparisons for jumps approached significance $(t \mathrm{~s}<1)$. Groups P48 and U48 circled more than did Group $S 48[t(42)=1.82, p<.05]$, and the greater number of circles by Group P48 than by Group U48 approached significance $[t(42)=1.61, p=.057]$. Neither 

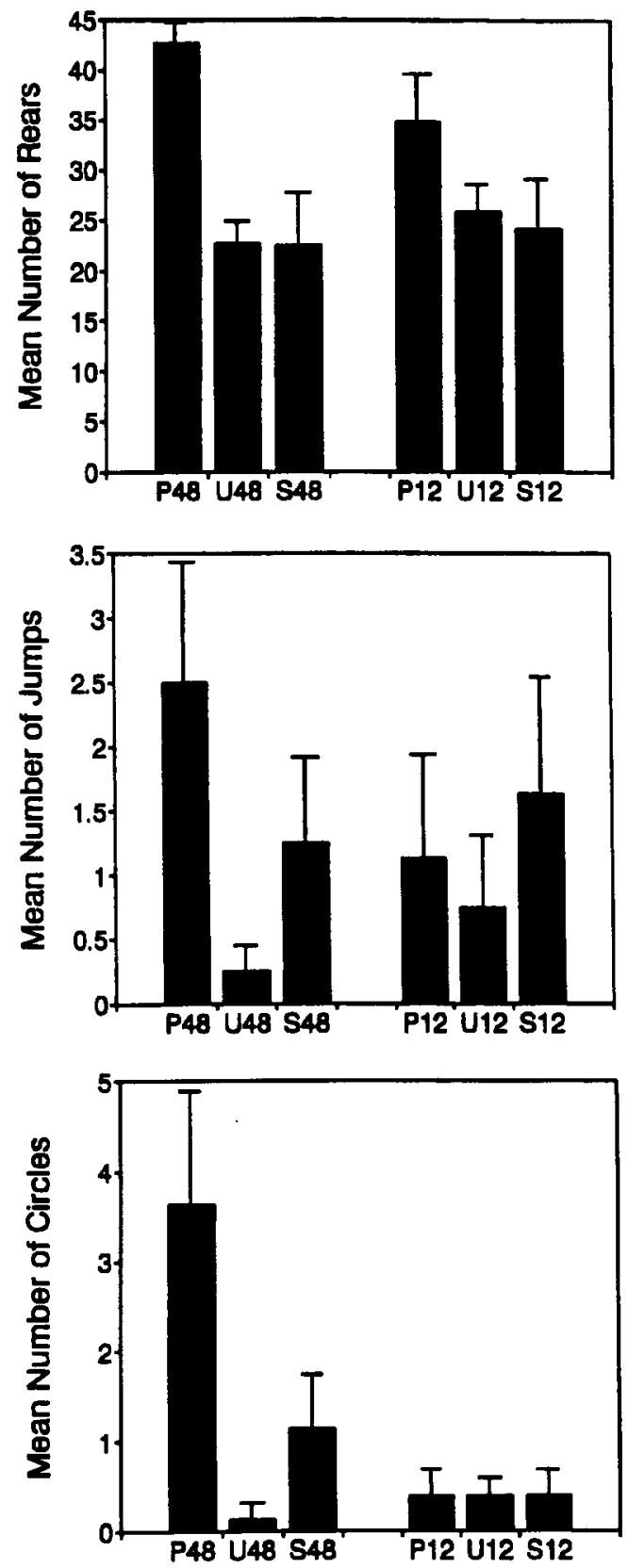

Figure 4. Mean withdrawal scores during the first withdrawal test in Experiment 2. Upper panel, rears; middle panel, jumps; lower panel, circles.

of the comparisons in the 12-h IDI condition approached reliability $(t \mathrm{~s}<1)$. None of the comparisons for wet $d o g$ shakes or genital licking were significant.

Figure 6 shows hot plate latencies during the tolerance retention test conducted $24 \mathrm{~h}$ later. The groups trained with morphine had significantly lower latencies than did the saline controls in the 48 -h IDI $[t(42)=2.67, p<$ $.01]$ and the 12 -h IDI $[t(42)=3.63, p<.01]$, but tolerance was not context specific in either IDI $(t s<1)$.

\section{Discussion}

Analgesic tolerance and three withdrawal signs (rears, jumps, and circles) were context specific in the 48-h IDI. In the 12-h condition, however, tolerance was not context specific, and only one withdrawal sign was controlled by the context. A week later, there was more context-specific withdrawal in Group P48 than in Group P12. Analgesic tolerance was retained, but it was not context specific in either IDI.

These results replicated the finding of Experiment 1 that the acquisition of context-specific tolerance and
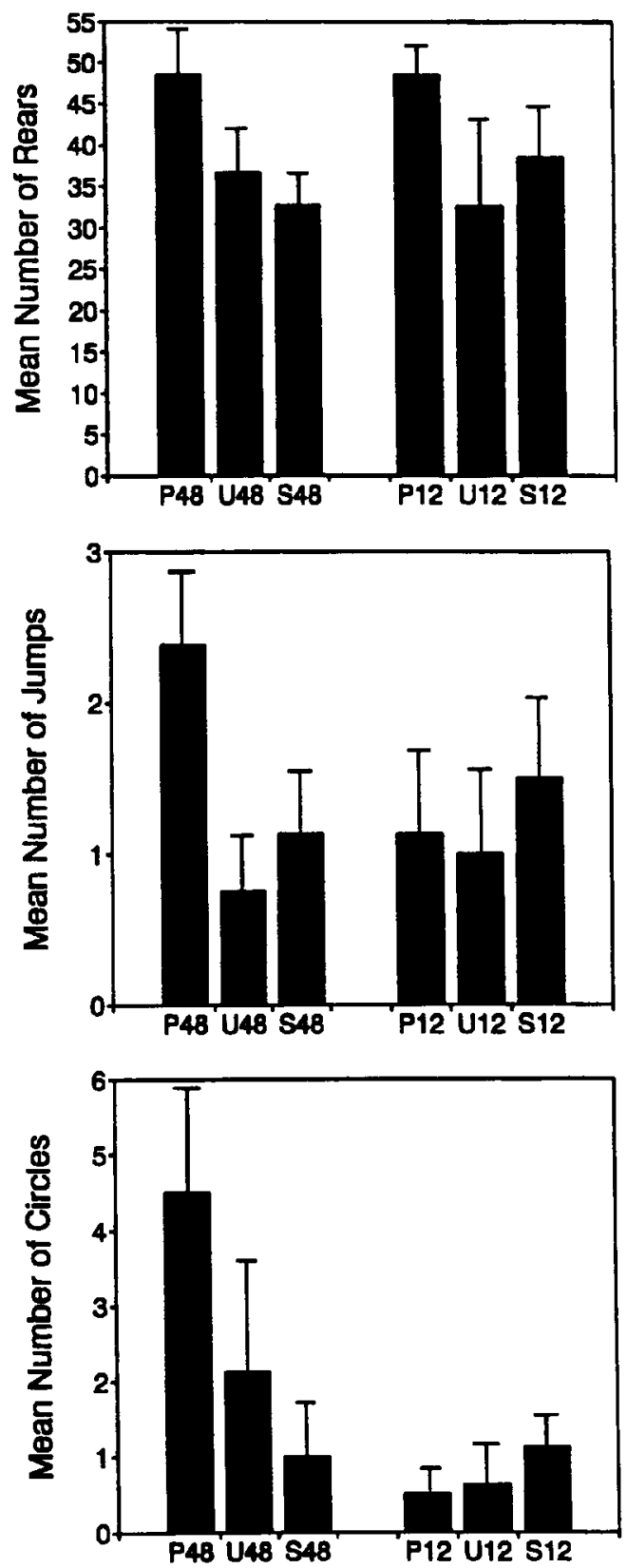

Figure 5. Retention of withdrawal in Experiment 2. Upper panel, rears; middle panel, jumps; lower panel, circles. 


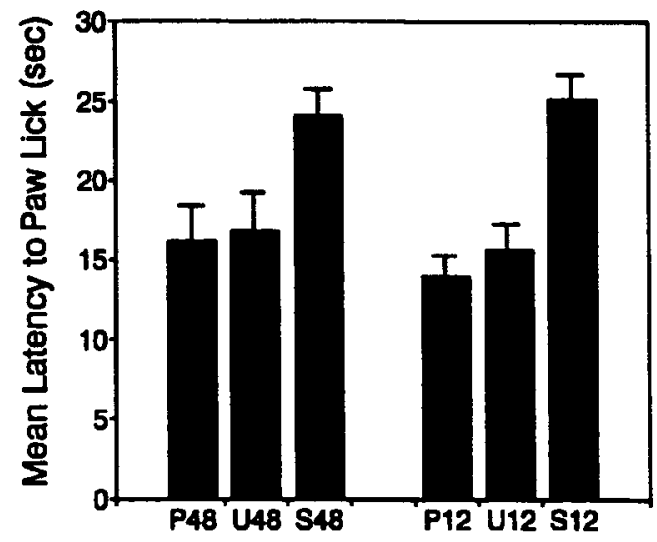

Figure 6. Mean latencies to lick a hind paw during the tolerance retention test in Experiment 2.

withdrawal is facilitated by spaced rather than by massed morphine administrations. It also showed that contextual control of withdrawal was retained for a somewhat longer time than that shown by previous demonstrations (Kelsey et al., 1990; Krank \& Perkins, 1993).

The finding that context-specific withdrawal and tolerance were similarly affected by the IDI supports the contention that they arise from a common process (Siegel, 1975, 1989). However, there were several instances in which the tolerance (hot plate) scores and the withdrawal signs did not exactly correspond. For example, the unpaired groups displayed no withdrawal, but substantial tolerance. Although it was not context specific, the retention of this tolerance suggests that it was associative; if so, some stimulus or stimuli must have mediated it. Several investigators have suggested that drug-onset cues may serve as CSs (McLaughlin, Dewey, \& Fanselow, 1991; Siegel, 1989; Walter \& Riccio, 1983). Although there is currently no evidence that morphine cues mediate morphine tolerance, there is evidence that alcohol can serve as a cue for itself and mediate tolerance (Greeley, Lê, Poulos, \& Cappell, 1984) and that pentobarbital cues can control morphine tolerance (Siegel, 1988). In the present experiments, if drug-onset cues mediated tolerance in the unpaired groups, no withdrawal signs would be expected during testing because these cues would not occur after a saline injection. Drug-onset cues could also explain the tolerance observed in Group P12, which was not context specific but was also retained. Indeed, some investigators have suggested that these cues overshadow contextual cues with a brief IDI (Walter \& Riccio, 1983). If this analysis is correct, the disruption of context-specific tolerance with a brief IDI is consistent with both the compensatory response model (Siegel, 1975, 1989) and the habituation model (Baker \& Tiffany, 1985).

Drug-onset cues cannot explain the contextual rearing seen in Group P12 and the corresponding lack of contextual tolerance. However, a precise correspondence between latency to paw lick and the withdrawal signs might not be expected. Different responses in other conditioning procedures are not exactly parallel. For example, a CS paired with a shock US elicits freezing and suppresses an operant baseline. Presumably, both freezing and conditioned suppression are mediated by the same central state-fear. Although there is ample evidence that these responses are similarly affected by various manipulations, (e.g., CS-US contingency, CS modality), they do not correspond exactly (Ayres, Axelrod, Mercker, Muchnik, \& Vigorito, 1985; Bouton \& Bolles, 1980). Although contextual tolerance and dependence did not precisely correspond, this experiment clearly shows that both were similarly affected by the IDI.

The failure to obtain retention of context-specific tolerance in Group P48 despite the retention of two withdrawal signs poses a more serious problem for the notion that these phenomena arise from a common process. Indeed, the lack of retention in Group P48 is puzzling because tolerance retention has been well documented (Dafters \& Odber, 1989; Siegel, 1975, 1977; Tiffany et al., 1991). In the present experiment, the lack of retention in Group P48 may have been an artifact of the testing procedure. There were two withdrawal tests between the first and second tolerance test, and each withdrawal test constituted an extinction trial for the paired groups, which would have diminished contextual control of tolerance. This explanation, while plausible, requires verification. Experiment 3 was performed to provide this. If contextual control of tolerance in Group P48 was due to the extinction that occurred during withdrawal testing, retention of contextual tolerance should be observed if the withdrawal tests are eliminated. The rats were trained in either the P48 or U48 conditions using a procedure identical to that of Experiment 2 and tested for tolerance 8 days after the last session. Saline controls were omitted because context-specific tolerance is defined by comparing the paired and unpaired groups.

\section{EXPERIMENT 3}

\section{Method}

\section{Subjects}

Sixteen male Sprague-Dawley rats were used. They weighed between 220 and $250 \mathrm{~g}$ and were maintained as in the previous experiments.

\section{Apparatus}

The DE and hot plate were those used in the first two experiments.

\section{Procedure}

Pretraining and tolerance development was identical to the 48-h IDI condition in Experiment 2. The rats received seven injections of morphine $(10 \mathrm{mg} / \mathrm{kg})$, spaced $48 \mathrm{~h}$ apart. The rats were randomly assigned to either the paired or unpaired groups. Eight days after the seventh session, all the rats received the usual dose of morphine in the DE and were tested on the hot plate at 20,40, and $60 \mathrm{~min}$ postinjection. 


\section{Results and Discussion}

Group P48 had substantially lower hot plate scores $(M=10.13 \mathrm{sec} ; S E M=0.94)$ than did Group U48 $(M=$ $15.57 \mathrm{sec} ; S E M=2.02$ ). This difference was significant $[t(14)=2.45, p<.05]$ and supports the explanation that the failure to observe retention of context-specific tolerance in the 48-h IDI in Experiment 2 was indeed an artifact of the testing procedure. Without the extinction trials produced by withdrawal testing, contextual control of tolerance was retained.

\section{GENERAL DISCUSSION}

These experiments clearly showed that contextual cues paired with morphine can elicit opiate withdrawal signs. Context-specific withdrawal was demonstrated with a dose that was substantially lower $(10 \mathrm{mg} / \mathrm{kg})$ than that used in previous studies $(50 \mathrm{mg} / \mathrm{kg}$ in Falls \& Kelsey, 1989, and Kelsey et al., 1990; $20 \mathrm{mg} / \mathrm{kg}$ in Krank \& Perkins, 1993) and was retained for a longer time (7 days) than that in previous demonstrations (Kelsey et al., 1990; Krank \& Perkins, 1993). In addition, these experiments showed that a procedure that produced context-specific withdrawal also resulted in contextspecific analgesic tolerance and is the only replication of Falls and Kelsey. Finally, these experiments showed that a procedure that reduced context-specific tolerance also reduced contextual control of withdrawal.

Although the measures of tolerance and withdrawal did not correspond precisely, both were similarly affected by the IDI manipulation. Although this manipulation is not a definitive test, the most parsimonious interpretation is that these phenomena were due to a common process (Siegel, 1975, 1989). These results could also be incorporated by the homeostatic model (Poulos \& Cappell, 1991), in which compensatory responses develop proportionally to the degree of drug-induced, homeostatic disturbance. The present experiments did not directly test this prediction of homeostatic theory, but it could easily account for these results by assuming that morphine-induced sedation was sufficiently aversive for hyperactivity to develop.

These results are also consistent with the tolerance model of Paletta and Wagner (1986), in which drug effects generally diminish because of habituation. In this model, compensatory responses also develop to drug effects that have a secondary, drug-opposite component that appears after the initial drug effect. For example, they showed that analgesia produced by morphine diminished and was replaced by a return to the predrug level of pain sensitivity. Sedation however, was followed by hyperactivity. This implies that conditioned hyperactivity and analgesic tolerance should develop in a similar but not exactly parallel fashion. Indeed, Paletta and Wagner (1986, Experiment 4) provided evidence for this by showing that either reducing or increasing the time spent in the context after a morphine injection disrupted context-specific analgesic tolerance. However, tolerance to sedation and conditioned hyperactivity were disrupted when the time in the context was increased, but not when it was decreased. This result is similar to the finding of Experiment 2 that context-specific analgesic tolerance was disrupted by the 12-h IDI, but rearing, an index of hyperactivity, was not.

In summary, these results provide support for the notion that these phenomena arise from a common process and are best explained by models that incorporate both associative tolerance and withdrawal. These results do not favor the compensatory response models (Siegel, 1975, 1989; Poulos \& Cappell, 1991) over the dualprocess model of Paletta and Wagner (1986). In addition, we cannot conclude that the withdrawal signs observed were compensatory responses. It seems plausible that rearing, circling, and jumping developed in response to sedation, but this remains speculative. Additional research is necessary to determine the precise relationship between conditioned tolerance, conditioned withdrawal signs, and the unconditional effects of morphine.

\section{REFERENCES}

Ayers, J. J. B., Axelrod, H., Mercker, E., MuchniK, F., \& Vigorito, M. (1985). Concurrent observations of barpress suppression and freezing: Effects of CS modality and on-line vs. off-line training upon posttrial behavior. Animal Learning \& Behavior, 13, 44-50.

BAKER, T. B., \& Tiffany, S. T. (1985). Morphine tolerance as habituation. Psychological Review, 92, 78-108.

Blasig, J., Hertz, A., Reinhold, K., \& Zieglgänsberger, S. (1973). Development of physical dependence on morphine in respect to time and dosage and quantification of the precipitated withdrawal syndrome in rats. Psychopharmacologia, 33, 19-38.

Bouton, M. E., \& Bolles, R. C. (1980). Conditioned fear assessed by freezing and by the suppression of three different baselines. Animal Learning \& Behavior, 8, 429-434.

DAFTERS, R., \& ODBER, J. (1989). Effects of dose, interdose interval, and drug-signal parameters on morphine analgesic tolerance: Implications for current theories of tolerance. Behavioral Neuroscience, 103, 1082-1090.

Falls, W. A., \& Kelsey, J. E. (1989). Procedures that produce context-specific tolerance to morphine in rats also produce context-specific withdrawal. Behavioral Neuroscience, 103, 842-849.

Goudie, A. J., \& Demellweek, C. (1986). Conditioning factors in drug tolerance. In S. R. Goldberg \& I. P. Stolerman (Eds.), Behavioral analysis of drug dependence (pp. 225-285). New York: Academic Press.

Greeley, J., LÊ, D. A., Poulos, C. X., \& Cappell, H. (1984). Alcohol is an effective cue in the conditional control of tolerance to alcohol. Psychopharmacology, 83, 159-162.

HAYS, W. L. (1981). Statistics (3rd ed.). New York: Holt, Rinehart \& Winston.

Hinson, R. W., \& Siegel, S. (1983). Anticipatory hyperexcitability and tolerance to the narcotizing effect of morphine in the rat. Behavioral Neuroscience, 97, 759-767.

Kelsey, J. E., Aranow, J. S., \& Matthews, R. T. (1990). Contextspecific morphine withdrawal in rats: Duration and effects of clonidine. Behavioral Neuroscience, 104, 704-710.

KESNER, R. P., \& COOK, D. G. (1983). The role of habituation and classical conditioning in the development of morphine tolerance. Behavioral Neuroscience, 97, 4-12.

Krank, M. D., Hinson, R. E., \& Siegel, S. (1981). Conditioned hyperalgesia is elicited by environmental signals of morphine. Behavioral \& Neural Biology, 32, 148-157.

Krank, M. D., \& Perkins, W. L. (1993). Conditioned withdrawal signs 
elicited by contextual cues for morphine administration. Psychobiology, 21, 113-119.

LINSEMAN, M. A. (1977). Naloxone-precipitated withdrawal as a function of the morphine-naloxone interval. Psychopharmacologia, 54, 159-164.

McLaughlin, C. R., Dewey, W. L., \& Fanselow, M. S. (1991). Shortand long-term factors in tolerance to morphine-induced antinociception: Single or multiple mechanisms? Psychobiology, 19, 217-222.

Paletta, M. S., \& Wagner, A. R. (1986). Development of context-specific tolerance to morphine: Support for a dual-process interpretation. Behavioral Neuroscience, 100, 611-623.

Poulos, C. X., \& CAPPELl, H. (1991). Homeostatic theory of drug tolerance: A general model of physiological adaptation. Psychological Review, 98, 390-408.

SCHNUR, P. (1992). Conditioned morphine withdrawal in the hamster. Psychopharmacology, 107, 517-522.

SIEGEL, S. (1975). Evidence from rats that morphine tolerance is a learned response. Journal of Comparative \& Physiological Psychology, 89, 498-506.

SIEGEL, S. (1977). Morphine tolerance acquisition as an associative process. Journal of Experimental Psychology: Animal Behavior Processes, 3, 1-13.

SIEGEL, S. (1978). Tolerance to the hyperthermic effect of morphine in the rat is a learned response. Journal of Comparative \& Physiological Psychology, 92, 1137-1149.

SIEGEL, S. (1988). State-dependent learning and morphine tolerance. Behavioral Neuroscience, 102, 228-232.

SIEGEL, S. (1989). Pharmacological conditioning and drug effects. In
A. J. Goudie \& M. Emmett-Oglesby (Eds.), Psychoactive drugs (pp. 115-180). Clifton, NJ: Humana.

SOBRERo, A. P., \& BouTON, M. E. (1989). Effects of stimuli present during oral morphine administration on withdrawal and subsequent consumption. Psychobiology, 17, 179-190.

TiffanY, S. T., \& BaKer, T. B. (1981). Morphine tolerance in rats: Congruence with a Pavlovian paradigm. Journal of Comparative \& Physiological Psychology, 95, 747-762.

Tiffany, S. T., \& Maude-Griffin, P. M. (1988). Tolerance to morphine in the rat: Associative and nonassociative effects. Behavioral Neuroscience, 102, 534-543.

Tiffany, S. T., Maude-Griffin, P. M., \& Drobes, D. J. (1991). Effect of interdose interval on the development of associative tolerance to morphine in the rat: A dose-response analysis. Behavioral Neuroscience, 105, 49-61.

WALTER, T. A., \& Riccio, D. C. (1983). Overshadowing effects in the stimulus control of morphine analgesic tolerance. Behavioral Neuroscience, 97, 658-662.

WikLer, A., \& PesCor, F. T. (1967). Classical conditioning of a morphine abstinence phenomenon, reinforcement of opioid-drinking behavior and "relapse" in morphine addicted rats. Psychopharmacologia, 10, 255-284.

Zellner, D. A., Dacanay, R. J., \& Riley, A. L. (1984). Opiate withdrawal: The result of conditioning or physiological mechanisms? Pharmacology, Biochemistry \& Behavior, 20, 175-180.

(Manuscript received July 19, 1993; revision accepted for publication July 7,1994 .) 